\title{
Birational sequences and the tropical Grassmannian
}

\author{
Lara Bossinger*
}

\begin{abstract}
We introduce iterated sequences for Grassmannians, a new class of Fang-Fourier-Littelmann's birational sequences and explain how they give rise to points in the tropical Grassmannian. For $\operatorname{Gr}\left(2, \mathbb{C}^{n}\right)$ we show that the associated valuations induce toric degenerations. We describe recursively the vertices of the corresponding Newton-Okounkov polytopes, which are particular vertices of a hypercube and hence integral. We show further that every toric degenerations of $\operatorname{Gr}\left(2, \mathbb{C}^{n}\right)$ constructed using the tropical Grassmannian due to Speyer and Sturmfels can be recovered by iterated sequences.
\end{abstract}

\section{Introduction}

Toric degenerations of Grassmannians or more generally flag and spherical varieties have been a vivid research topic during the last 20 years. A novel framework, called birational sequences, stemming from representation theory was given by Fang, Fourier and Littelmann in [FFL17]. Within this framework, we introduce and analyze new birational sequences for Grassmannians and discover surprising connections to the tropical Grassmannian by Speyer-Sturmfels [SS04].

To be more precise we need to introduce a bit of notation. A toric degeneration of a projective variety $X$ is a flat morphism $\pi: \mathcal{X} \rightarrow \mathbb{A}^{d}$ with generic fiber $\pi^{-1}(t)$ for $t \neq 0$ isomorphic to $X$ and $\pi^{-1}(0)$ a projective toric variety. Consider $S L_{n}$ over $\mathbb{C}$ with Borel subgroup $B$ of upper triangular matrices. Let $P_{k}$ be the parabolic subgroup of block-upper triangular matrices with blocks of size $k \times k$ and $(n-k) \times(n-k)$. In particular, the Grassmannian $\operatorname{Gr}\left(k, \mathbb{C}^{n}\right)$ is isomorphic to $S L_{n} / P_{k}$. Denote its dimension by $d$. Further, let $U^{-}$be lower triangular matrices with 1's along the diagonal. The root system of type $A_{n-1}$ is $R=\left\{\varepsilon_{i}-\varepsilon_{j}\right\}_{i \neq j}$ where $\left\{\varepsilon_{i}\right\}_{i}$ is the standard basis of $\mathbb{R}^{n}$. For every positive root $\beta \in R^{+}$we have the one-parameter root subgroup $U_{-\beta} \subset U^{-}$. In this setting the notion of birational sequences due to Fang, Fourier and Littelmann [FFL17] applies: a sequence $S=\left(\beta_{1}, \ldots, \beta_{d}\right)$ of positive roots is called birational for $\operatorname{Gr}\left(k, \mathbb{C}^{n}\right)$ if the multiplication map

$$
U_{-\beta_{1}} \times \cdots \times U_{-\beta_{d}} \rightarrow U^{-}
$$

has image birational to $\operatorname{Gr}\left(k, \mathbb{C}^{n}\right)$. Birational sequences give rise to coordinates on the Grassmannian. These can be used to define valuations on the homogeneous coordinate ring. The construction allows to compute certain values of the valuations explicitly via representations of the Lie algebra $\mathfrak{s l}_{n}$. If the valuation has finitely generated value semi-group it induces a toric degeneration of the Grassmannian by [And13]. Their setting unifies many known constructions of toric degenerations in representation theory, such as [GL96, Cal02, AB04, FFL11]. However, besides the previously known cases few new birational sequences were discovered so far. A central question is whether toric degenerations constructed from tropical geometry (see e.g. [SS04] for the case of Grassmannians) or cluster algebras (see e.g. [RW19]) can be recovered using birational sequences.

In this paper we study birational sequences that do not correspond to the previously known ones. One way to construct them is provided by the following central Lemma (see also Lemma 2). It allows to obtain a birational sequences for $\operatorname{Gr}\left(k, \mathbb{C}^{n+1}\right)$ from a birational sequence for $\operatorname{Gr}\left(k, \mathbb{C}^{n}\right)$.

\footnotetext{
*Supported by "Programa de Becas Posdoctorales en la UNAM 2018" Instituto de Matemáticas, Universidad Nacional Autónoma de México.
} 
Lemma. Let $S=\left(\beta_{1}, \ldots, \beta_{d}\right)$ be a birational sequence for $\operatorname{Gr}\left(k, \mathbb{C}^{n}\right)$ and choose $i_{1}, \ldots, i_{k}$ pairwise differently from $\{1, \ldots, n\}$. Then the following is a birational sequence for $\operatorname{Gr}\left(k, \mathbb{C}^{n+1}\right)$ :

$$
S^{\prime}=\left(\varepsilon_{i_{1}}-\varepsilon_{n+1}, \ldots, \varepsilon_{i_{k}}-\varepsilon_{n+1}, \beta_{1}, \ldots, \beta_{d}\right) .
$$

Inspired by the Lemma we define a new class of birational sequences: a birational sequence $S$ for $\operatorname{Gr}\left(k, \mathbb{C}^{n}\right)$ constructed from a birational sequence for $\operatorname{Gr}\left(k, \mathbb{C}^{k+1}\right)$ by applying the Lemma $n-k-1$ many times is called iterated (see Definition 11).

Focusing on iterated sequences for $\operatorname{Gr}\left(2, \mathbb{C}^{n}\right)$, we show further that the corresponding valuations have the necessary property to induce toric degenerations (relying on a result of [Bos20]). Fixing the Plücker embedding of $\operatorname{Gr}\left(2, \mathbb{C}^{n}\right)$ the corresponding Newton-Okounkov polytopes are in fact integral polytopes inside the hypercube ${ }^{1}$ in $\mathbb{R}_{>0}^{2(n-2)}$ (see Corollary 2). The vertices are given by the images of the valuation on Plücker coordinates, which in this case form a Khovanskii basis.

Moreover, we identify iterated sequences with maximal cones in $\operatorname{trop}\left(\operatorname{Gr}\left(k, \mathbb{C}^{n}\right)\right)$, the tropical Grassmannian [SS04]. For $k=2$ we give the explicit Algorithm 1 to do so. For an iterated sequence $S$ denote by $C_{S} \subset \operatorname{trop}\left(\operatorname{Gr}\left(2, \mathbb{C}^{n}\right)\right)$ the output of the algorithm. We say two toric degenerations of a projective variety $X$ are equivalent if their special fibers are isomorphic toric varieties. Our main results (Theorems $1 \& 2$ ) can be summarized as follows:

Theorem. Let $S$ be an iterated sequence for $\operatorname{Gr}\left(k, \mathbb{C}^{n}\right)$. Then there exists a maximal cone $C \subset$ $\operatorname{trop}\left(\operatorname{Gr}\left(k, \mathbb{C}^{n}\right)\right)$ such that the toric degeneration of $\operatorname{Gr}\left(k, \mathbb{C}^{n}\right)$ induced by $S$ is equivalent to the one given by the maximal cone $C \subset \operatorname{trop}\left(\operatorname{Gr}\left(k, \mathbb{C}^{n}\right)\right)$.

Moreover, for $k=2$ the cone $C$ equals the cone $C_{S}$ which is the output Algorithm 1. Conversely, for every maximal cone $C \subset \operatorname{trop}\left(\operatorname{Gr}\left(2, \mathbb{C}^{n}\right)\right)$ there exists an iterated sequence $S$ such that the induced toric degenerations of $C$ and $S$ are equivalent.

Remark 1. Combining with the results of $\left[\mathrm{BFF}^{+} 18\right]$, where toric degenerations of $\operatorname{Gr}\left(2, \mathbb{C}^{n}\right)$ from plabic graphs are studied, our main result implies that iterated sequences not only recover all possible toric degenerations of $\operatorname{Gr}\left(2, \mathbb{C}^{n}\right)$ constructed using the tropical Grassmannian, but also all those constructed using plabic graphs (as in [RW19]). Said differently, up to equivalence of toric degenerations the following sets are in one-to-one correspondence:

$$
\left\{\begin{array}{c}
\text { toric degenerations } \\
\text { of } \operatorname{Gr}\left(2, \mathbb{C}^{n}\right) \text { from } \\
\text { iterated sequences }
\end{array}\right\} \stackrel{\text { Theorems } 1 \& 2}{\longleftrightarrow}\left\{\begin{array}{c}
\text { toric degenerations } \\
\text { of } \mathrm{Gr}\left(2, \mathbb{C}^{n}\right) \text { from } \\
\operatorname{trop}\left(\mathrm{Gr}\left(2, \mathbb{C}^{n}\right)\right)
\end{array}\right\} \stackrel{\left[\mathrm{BFF}^{+} 18\right]}{\longleftrightarrow}\left\{\begin{array}{c}
\text { toric degenerations } \\
\text { of } \mathrm{Gr}\left(2, \mathbb{C}^{n}\right) \text { from } \\
\text { plabic graphs }
\end{array}\right\}
$$

We analyze the combinatorial structure of iterated sequences in terms of trivalent trees with $n$ leaves that are in correspondence with maximal cones of $\operatorname{trop}\left(\operatorname{Gr}\left(2, \mathbb{C}^{n}\right)\right)$. Let $\mathcal{T}$ be the infinite graph with vertices corresponding to (unlabelled) trivalent trees. Two vertices $\mathrm{T}_{1}, \mathrm{~T}_{2}$ of $\mathcal{T}$ are connected by an directed edge $\mathrm{T}_{1} \rightarrow \mathrm{T}_{2}$, if $\mathrm{T}_{2}$ can be obtained from $\mathrm{T}_{1}$ by adding a leaf-edge (see Figure 1). The unique source of $\mathcal{T}$ is the trivalent tree with three leaves. Algorithm 1 yields the following corollary:

Corollary. Every iterated sequence $S$ for $\operatorname{Gr}\left(2, \mathbb{C}^{n}\right)$ yields a unique path from the source of $\mathcal{T}$ to the trivalent tree with $n$ leaves corresponding to the equivalence class $^{2}$ of the maximal cone $C_{S} \subset$ $\operatorname{trop}\left(\operatorname{Gr}\left(2, \mathbb{C}^{n}\right)\right)$.

The paper is structured as follows: after setting up the notation in $\S 2$ we recall necessary notions on valuations in $\S 2.1$. Reminders on tropical geometry can be found in $\$ 2.2$. In $\S 3$ we introduce iterated (birational) sequences for Grassmannians and recall the construction of the associated valuations due to [FFL17]. In $\S 4$ we focus on iterated sequences for $\operatorname{Gr}\left(2, \mathbb{C}^{n}\right)$ and prove the main result.

\footnotetext{
${ }^{1}$ We consider the hypercube as the convex hull of all elements in $\{0,1\}^{2(n-2)} \subset \mathbb{R}_{\geq 0}^{2(n-2)}$.

${ }^{2}$ Equivalence classes are considered with respect to the action of the symmetric group $S_{n}$ on the Plücker coordinates, see $\S 2.2$ below Remark 2
} 


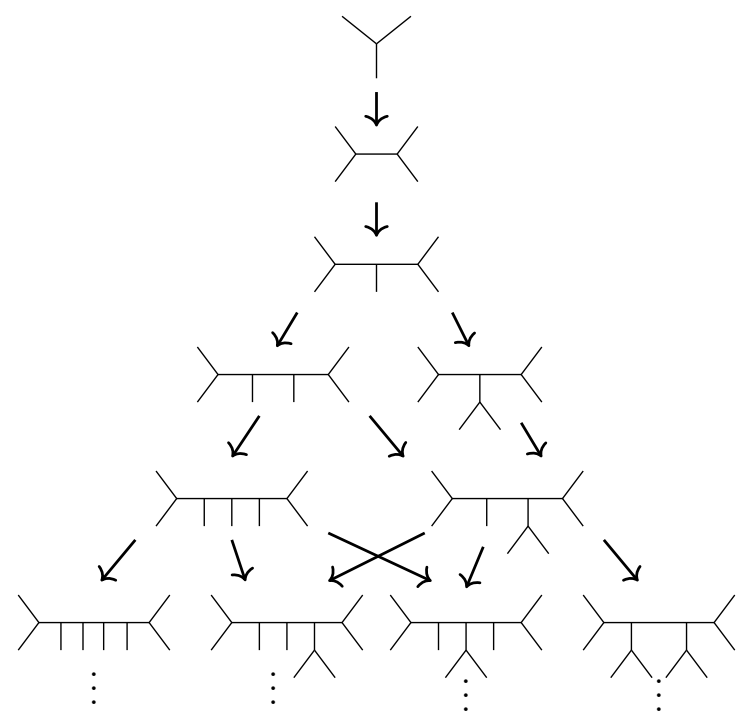

Figure 1: The tree graph $\mathcal{T}$ from level (\# \#f leaves) 3 to 8.

Acknowledgements: The result of this paper is part of my PhD thesis [Bos18] supervised by Peter Littelmann at the University of Cologne. I am grateful for his advice and support throughout my $\mathrm{PhD}$. Further, I would like to thank Xin Fang, Ghislain Fourier and Alfredo Nájera Chávez for inspiring and helpful discussions and an anonymous referee for asking a question that lead to Corollary 3.

\section{Notation}

Let $I_{k, n}$ be the set of ordered integer sequences $\underline{i}=\left(i_{1}<\cdots<i_{k}\right)$ with $1 \leq i_{1}$ and $i_{k} \leq n$. We consider the Grassmannian $\operatorname{Gr}\left(k, \mathbb{C}^{n}\right)$ of $k$-dimensional subspaces in $\mathbb{C}^{n}$ with its Plücker embedding. It is the vanishing of the Plücker ideal $\mathcal{I}_{k, n} \subset \mathbb{C}\left[p_{\underline{j}} \mid \underline{j} \in I_{k, n}\right]$ in the polynomial ring on Plücker variables $p_{\underline{j}}$. To describe $\mathcal{I}_{k, n}$ more explicitly we need some more notation.

Denote $\{1, \ldots, n\}$ by $[n]$. Consider $\underline{j}=\left(j_{1}<\cdots<j_{k+1}\right) \in I_{k+1, n}$ and $j_{s} \in \underline{j}$, i.e. $1 \leq s \leq k+1$. Then $\underline{j} \backslash j_{s}$ denotes the sequence in $I_{k, n}$ obtained by removing $j_{s}$ from $\underline{j}$. For $\underline{i}=\left(i_{1}<\cdots<i_{k-1}\right) \in$ $I_{k-1, n}$ and $j \in[n]$ define

$$
\underline{i} \cup j:=\left(i_{1}<\cdots<i_{r}<j<i_{r+1}<\cdots<i_{k-1}\right) .
$$

Set $\ell(\underline{i}, j):=k-(r+1)$. Note that if $j=i_{l}$ for some $1 \leq l \leq k-1$ then $\underline{i} \cup j \notin I_{k, n}$, in this case we set $p_{\underline{i}} \cup j=0$. The Plücker ideal $\mathcal{I}_{k, n}$ is generated by elements of form

$$
R_{\underline{i}, \underline{j}}:=\sum_{s=1}^{k+1}(-1)^{\ell\left(\underline{i}, j_{s}\right)} p_{\underline{i} \cup j_{s}} p_{\underline{j} \backslash j_{s}},
$$

for $\underline{i} \in I_{k-1, n}$ and $\underline{j} \in I_{k+1, n}$ (see e.g. [LB15, §4.2.2]). For $k=2$ we simplify notation by $p_{i j}:=p_{(i<j)}$ for $(i<j) \in I_{2, n}$. Then the Plücker relations are of form:

$$
R_{r, s, u, v}:=R_{(r),(s<u<v)}=p_{r s} p_{u v}-p_{r u} p_{s v}+p_{r v} p_{s u} \in \mathbb{C}\left[p_{i j}\right]_{i<j},
$$

with $1 \leq r<s<u<v \leq n$. Let $A_{k, n}:=\mathbb{C}\left[p_{\underline{j}}\right]_{\underline{j} \in I_{k, n}} / \mathcal{I}_{k, n} \cong \mathbb{C}\left[\operatorname{Gr}\left(k, \mathbb{C}^{k}\right)\right]$, which is the homogeneous coordinate ring of the Grassmannian. The cosets $\bar{p}_{\underline{j}} \in A_{k, n}$ are called Plücker coordinates. 
We recall some standard facts about Lie algebras, algebraic groups and their representations that will be used throughout the rest of the paper. Let $\mathfrak{s l}_{n}$ be the Lie algebra corresponding to $S L_{n}$ and fix a Cartan decomposition $\mathfrak{s l}_{n}=\mathfrak{n}^{-} \oplus \mathfrak{h} \oplus \mathfrak{n}^{+}$. The Cartan subalgebra $\mathfrak{h}$ consists of diagonal traceless matrices, and $\mathfrak{n}^{-}$(resp. $\mathfrak{n}^{+}$) of strictly lower (resp. upper) triangular matrices.

In the type $\mathrm{A}_{n-1}$ root system $R=\left\{\varepsilon_{i}-\varepsilon_{j}\right\}_{i \neq j \in[n]} \subset \mathbb{R}^{n}$ we have positive roots $R^{+}=\left\{\varepsilon_{i}-\varepsilon_{j}\right\}_{i<j}$ and simple roots $\left\{\varepsilon_{i}-\varepsilon_{i+1}\right\}_{i=1, \ldots, n-1}$. Further, set $R_{k}^{+}:=\left\{\varepsilon_{i}-\varepsilon_{j} \mid 1 \leq i \leq k \leq j \leq n\right\}$. For every positive root $\varepsilon_{i}-\varepsilon_{j}$ we have a root operator $f_{i, j} \in \mathfrak{n}^{-}$: it is the elementary matrix with only non-zero entry being 1 in the $(j, i)$-position.

Let $\Lambda$ denote the weight lattice, generated by fundamental weights $\omega_{1}, \ldots, \omega_{n-1}$. The $k$ th fundamental representation of $\mathfrak{s l}_{n}$ is $V\left(\omega_{k}\right)=\Lambda^{k} \mathbb{C}^{n}$. It is cyclically generated by a highest weight vector $v_{\omega_{k}}$ over the universal enveloping algebra $U\left(\mathfrak{n}^{-}\right)$.

Example 1. For $V\left(\omega_{2}\right)$ fix the basis $\left\{e_{i} \wedge e_{j} \mid 1 \leq i<j \leq n\right\}$. Then the action of $\mathfrak{n}^{-}$is given by

$$
f_{i, j} \cdot\left(e_{k} \wedge e_{l}\right)=f_{i, j} \cdot e_{k} \wedge e_{l}+e_{k} \wedge f_{i, j} \cdot e_{l}=\left\{\begin{array}{cc}
e_{j+1} \wedge e_{l}, & \text { if } k=i, \\
e_{k} \wedge e_{j+1}, & \text { if } l=i, \\
0, & \text { otherwise. }
\end{array}\right.
$$

We choose $e_{1} \wedge e_{2}$ as the highest weight vector $v_{\omega_{2}}$.

Identifying the Grassmannian with the quotient $S L_{n} / P_{k}$, the Plücker embedding can be written as $\operatorname{Gr}\left(k, \mathbb{C}^{n}\right) \hookrightarrow \mathbb{P}\left(V\left(\omega_{k}\right)\right)$. For $r \geq 1$ let $V\left(r \omega_{k}\right)^{*}$ denote the vector space dual to $V\left(r \omega_{k}\right)$. We obtain $A_{k, n} \cong \bigoplus_{r \geq 0} V\left(r \omega_{k}\right)^{*}$ and the Plücker coordinate $\bar{p}_{\underline{j}} \in A_{k, n}$ for $\underline{j}=\left(j_{1}<\cdots<j_{k}\right) \in I_{k, n}$ is the dual basis element $\left(e_{j_{1}} \wedge \cdots \wedge e_{j_{k}}\right)^{*} \in V\left(\omega_{k}\right)^{*}$.

Let $U_{k}^{-} \subset U^{-}$be the subgroup generated by elements $\exp \left(z f_{i, j}\right)=\mathbb{1}+z f_{i, j}$, where $\varepsilon_{i}-\varepsilon_{j} \in R_{k}^{+}$. Then $U_{k}^{-}$is open and dense in $S L_{n} / P_{k} \cong \operatorname{Gr}\left(k, \mathbb{C}^{n}\right)$. Hence, we have Plücker coordinates on $U_{k}^{-}: \bar{p}_{\underline{j}}$ is the $k \times k$-minor on columns $[k]$ and rows $\underline{j}$. Moreover, we have an isomorphism of the fields of rational functions $\mathbb{C}\left(\operatorname{Gr}\left(k, \mathbb{C}^{n}\right)\right) \cong \mathbb{C}\left(U_{k}^{-}\right)$.

\subsection{Valuations}

We recall basic notions on valuations and Newton-Okounkov polytopes as presented in [KK12].

Consider $A=\mathbb{C}\left[x_{1}, \ldots, x_{n}\right] / I$ with $I$ a homogeneous ideal and kernel of $\pi: \mathbb{C}\left[x_{1}, \ldots, x_{n}\right] \rightarrow A$. Denote $\bar{x}_{i}:=\pi\left(x_{i}\right)$. The standard grading on the polynomial ring induces a positive grading on $A=\bigoplus_{i \geq 0} A_{i}$. Let $d$ be the Krull-dimension of $A$. Additionally, fix a linear order $\prec$ on the additive abelian group $\mathbb{Z}^{d}$.

Definition 1. A map $\mathfrak{v}: A \backslash\{0\} \rightarrow\left(\mathbb{Z}^{d}, \prec\right)$ is a valuation, if it satisfies for all $f, g \in A \backslash\{0\}$ and $c \in \mathbb{C}^{*}$

(i) $\mathfrak{v}(f+g) \succeq \min \{\mathfrak{v}(f), \mathfrak{v}(g)\}$,

(ii) $\mathfrak{v}(f g)=\mathfrak{v}(f)+\mathfrak{v}(g)$ and

(iii) $\mathfrak{v}(c f)=\mathfrak{v}(f)$.

Let $\mathfrak{v}: A \backslash\{0\} \rightarrow\left(\mathbb{Z}^{d}, \prec\right)$ be a valuation. The image $\{\mathfrak{v}(f) \mid f \in A \backslash\{0\}\} \subset \mathbb{Z}^{d}$ forms an additive semi-group. We denote it by $S(A, \mathfrak{v})$ and refer to it as the value semi-group. The rank of the valuation is the rank of the sublattice generated by $S(A, \mathfrak{v})$ in $\mathbb{Z}^{d}$. We are interested in valuations of full rank, i.e. $\operatorname{rank}(\mathfrak{v})=d$.

One naturally defines a $\mathbb{Z}^{d}$-filtration on $A$ by $F_{\mathfrak{v} \succeq a}:=\{f \in A \backslash\{0\} \mid \mathfrak{v}(f) \succeq a\} \cup\{0\}$ (and similarly $\left.F_{\mathfrak{v} \succ a}\right)$. The associated graded algebra is

$$
\operatorname{gr}_{\mathfrak{v}}(A):=\bigoplus_{a \in \mathbb{Z}^{d}} F_{\mathfrak{v} \succeq a} / F_{\mathfrak{v} \succ a} .
$$

If the filtered components $F_{\mathfrak{v} \succeq a} / F_{\mathfrak{v} \succ a}$ are at most one-dimensional for all $a \in \mathbb{Z}^{d}$, we say $\mathfrak{v}$ has onedimensional leaves. By [KM19, Theorem 2.3] the full-rank assumption on $\mathfrak{v}$ implies that $\mathfrak{v}$ has onedimensional leaves. In particular, by [BG09, Remark 4.13] we then have an isomorphism $\mathbb{C}[S(A, \mathfrak{v})] \cong$ $\operatorname{gr}_{\mathfrak{v}}(A)$. To define a $\mathbb{Z}_{\geq 0}$-filtration on $A$ induced by $\mathfrak{v}$ we make use of the following lemma: 
Lemma. ( [Cal02, Lemma 3.2]) Let $S$ be a finite subset of $\mathbb{Z}^{d}$. Then there exists a linear form $e: \mathbb{Z}^{d} \rightarrow \mathbb{Z}_{\geq 0}$ such that for all $\mathbf{m}, \mathbf{n} \in S$ we have $\mathbf{m} \prec \mathbf{n} \Rightarrow e(\mathbf{m})>e(\mathbf{n})$ (note the switch!).

Assume $S(A, \mathfrak{v})$ is finitely generated, more precisely assume $S(A, \mathfrak{v})$ is generated by $\mathfrak{v}\left(\bar{x}_{1}\right), \ldots, \mathfrak{v}\left(\bar{x}_{n}\right)$. In this case $\left\{\bar{x}_{1}, \ldots, \bar{x}_{n}\right\}$ is called a Khovanskii basis ${ }^{3}$ for $(A, \mathfrak{v})$. Now choose a linear form as in the lemma for $S$ being the set of generators. We construct a $\mathbb{Z}_{\geq 0}$-filtration on $A$ by $F_{\leq m}:=\{f \in A \backslash\{0\} \mid$ $e(\mathfrak{v}(f)) \leq m\} \cup\{0\}$ for $m \in \mathbb{Z}_{\geq 0}$. Define similarly $F_{<m}$. The associated graded algebra satisfies

$$
\operatorname{gr}_{\mathfrak{v}}(A) \cong \bigoplus_{m \geq 0} F_{\leq m} / F_{<m}
$$

For $f \in A \backslash\{0\}$ denote by $\bar{f}$ its image in the quotient $F_{\leq e(\mathfrak{v}(f))} / F_{<e(\mathfrak{v}(f))}$, hence $\bar{f} \in \operatorname{gr}_{\mathfrak{v}}(A)$. We obtain a family of $\mathbb{C}$-algebras containing $A$ and $\operatorname{gr}_{\mathfrak{v}}(A)$ as fibers (see e.g. [And13, Proposition 5.1]) that can be defined as follows:

Definition 2. The Rees algebra associated with the valuation $\mathfrak{v}$ and the filtration $\left\{F_{\leq m}\right\}_{m}$ is the flat $\mathbb{C}[t]$-subalgebra of $A[t]$ defined as

$$
R_{\mathfrak{v}, e}:=\bigoplus_{m \geq 0}\left(F_{\leq m}\right) t^{m} .
$$

It has the properties that $R_{\mathfrak{v}, e} / t R_{\mathfrak{v}, e} \cong \operatorname{gr}_{\mathfrak{v}}(A)$ and $R_{\mathfrak{v}, e} /(1-t) R_{\mathfrak{v}, e} \cong A$. In particular, it defines a flat family over $\mathbb{A}^{1}$ (the coordinate on $\mathbb{A}^{1}$ given by $t$ ). The generic fiber is isomorphic to $\operatorname{Proj}(A)$ and the special fiber is the toric variety $\operatorname{Proj}\left(\operatorname{gr}_{\mathfrak{v}}(A)\right)$, where Proj is taken with respect to the $\mathbb{Z}_{\geq 0}$-grading on $A$.

Introduced by Lazarsfeld-Mustaţă [LM09] and Kaveh-Khovanskii [KK12] we recall the definition of Newton-Okounkov body.

Definition 3. Let $\mathfrak{v}: A \backslash\{0\} \rightarrow\left(\mathbb{Z}^{d}, \prec\right)$ be a valuation of full rank. The Newton-Okounkov body is

$$
\Delta(A, \mathfrak{v}):=\overline{\operatorname{conv}\left(\bigcup_{i>0}\left\{\mathfrak{v}(f) / i \mid 0 \neq f \in A_{i}\right\}\right)}
$$

Anderson showed in [And13] that if $\operatorname{gr}_{\mathfrak{v}}(A)$ is finitely generated, then $\Delta(A, \mathfrak{v})$ is a rational polytope. Moreover, it is the polytope associated to the normalization of the toric variety $\operatorname{Proj}\left(\operatorname{gr}_{\mathfrak{v}}(A)\right)$.

The aim of [Bos20] is to give a criterion for when a valuation induces toric degeneration. Using Anderson's result, this translates to giving a criterion for when the corresponding value semi-group is finitely generated. We state the criterion [Bos20, Lemma 3 and Theorem 1] below and use it to prove our main theorem in $\S 4$. It uses the notion of initial ideals, that are defined as follows. For $u \in \mathbb{Z}_{\geq 0}^{n}$ let $x^{u}$ to denote the monomial $x_{1}^{u_{1}} \cdots x_{n}^{u_{n}} \in \mathbb{C}\left[x_{1}, \ldots, x_{n}\right]$.

Definition 4. Let $f=\sum a_{u} x^{u} \in \mathbb{C}\left[x_{1}, \ldots, x_{n}\right]$ and fix an element $w \in \mathbb{R}^{n}$ (resp. $M \in \mathbb{Z}^{d \times n}$ ). Then the initial form of $f$ with respect to $w$ (resp. $M$ ) is

$$
\operatorname{in}_{w}(f):=\sum_{w \cdot u \text { minimal }} a_{u} x^{u}, \quad \text { resp. } \quad \operatorname{in}_{M}(f):=\sum_{M m=\min \prec\left\{M u \mid a_{u} \neq 0\right\}} a_{m} x^{m} .
$$

Let $I \subset \mathbb{C}\left[x_{1}, \ldots, x_{n}\right]$ be an ideal. Then its initial ideal with respect to $w \in \mathbb{R}^{n}$ (resp. $M \in \mathbb{Z}^{d \times n}$ ) is defined as

$$
\operatorname{in}_{w}(I):=\left\langle\operatorname{in}_{w}(f) \mid f \in I\right\rangle, \quad \text { resp. } \quad \operatorname{in}_{M}(I):=\left\langle\operatorname{in}_{M}(f) \mid f \in I\right\rangle .
$$

Definition 5. Given a valuation $\mathfrak{v}: A \backslash\{0\} \rightarrow\left(\mathbb{Z}^{d}, \prec\right)$ define the weighting matrix of $\mathfrak{v}$ by

$$
M_{\mathfrak{v}}:=\left(\mathfrak{v}\left(\bar{x}_{1}\right), \ldots, \mathfrak{v}\left(\bar{x}_{n}\right)\right) \in \mathbb{Z}^{d \times n} .
$$

That is, the columns of $M_{\mathfrak{v}}$ are given by the images $\mathfrak{v}\left(\bar{x}_{i}\right)$ for $i \in[n]$.

\footnotetext{
${ }^{3}$ This term was introduced in [KM19] generalizing the notion of SAGBI basis
} 
Theorem. ( [Bos20, Theorem 1]) Let $\mathfrak{v}: A \backslash\{0\} \rightarrow\left(\mathbb{Z}^{d}, \prec\right)$ be a full-rank valuation with $M_{\mathfrak{v}} \in \mathbb{Z}^{d \times n}$ the weighting matrix of $\mathfrak{v}$. Then

$$
S(A, \mathfrak{v}) \text { is generated by }\left\{\mathfrak{v}\left(\bar{x}_{i}\right)\right\}_{i \in[n]} \quad \Leftrightarrow \quad \operatorname{in}_{M_{\mathfrak{v}}}(I) \text { is prime and } M_{\mathfrak{v}} \text { is of full rank. }
$$

In this case $\left\{\bar{x}_{1}, \ldots, \bar{x}_{n}\right\}$ forms a Khovanskii basis for $(A, \mathfrak{v})$ and $\Delta(A, \mathfrak{v})=\operatorname{conv}\left(\mathfrak{v}\left(\bar{x}_{i}\right) \mid i \in[n]\right)$.

In particular, the theorem (resp. its proof) implies that if $\operatorname{in}_{M_{\mathfrak{v}}}(I)$ is prime, we have $\operatorname{gr}_{\mathfrak{v}}(A) \cong$ $\mathbb{C}\left[x_{1}, \ldots, x_{n}\right] / \operatorname{in}_{M_{\mathfrak{v}}}(I)$. To make a connection to tropical geometry, more precisely the tropical Grassmannian, we rely on the following Lemma.

Lemma. ( [Bos20, Lemmata 2\&3]) Let $\mathfrak{v}: A \backslash\{0\} \rightarrow\left(\mathbb{Z}^{d}, \prec\right)$ be a full-rank valuation and $M_{\mathfrak{v}}$ the associated weighting matrix. Then $\operatorname{in}_{M_{\mathfrak{v}}}(I)$ is monomial-free. Moreover, there exists $w \in \mathbb{Z}^{n}$ with $\operatorname{in}_{w}(I)=\operatorname{in}_{M_{\mathfrak{v}}}(I)$.

\subsection{Tropical Geometry}

We recall basic notions on tropical geometry, in particular on the tropical Grassmannian. For details we refer to [MS15] and [SS04]. For $u \in \mathbb{Z}^{n}$ as before we denote $x^{u}=x_{1}^{u_{1}} \cdots x_{n}^{u_{n}} \in \mathbb{C}\left[x_{1}^{ \pm 1}, \ldots, x_{n}^{ \pm 1}\right]$.

Definition 6. Let $f=\sum a_{u} x^{u} \in \mathbb{C}\left[x_{1}^{ \pm 1}, \ldots, x_{n}^{ \pm 1}\right]$. The tropicalization of $f$ is the function $f^{\text {trop }}$ : $\mathbb{R}^{n} \rightarrow \mathbb{R}$ given by

$$
f^{\operatorname{trop}}(w):=\min \left\{w \cdot u \mid u \in \mathbb{Z}^{n} \text { and } a_{u} \neq 0\right\} .
$$

If $w-v=(m, \ldots, m)$, for some $v, w \in \mathbb{R}^{n}$ and $m \in \mathbb{R}$, we have that the minimum in $f^{\text {trop }}(w)$ and $f^{\operatorname{trop}}(v)$ is achieved for the same $u \in \mathbb{Z}^{n}$ with $a_{u} \neq 0$.

Definition 7. ( $\left[\mathrm{MS} 15\right.$, Definitions 3.1.1/2]) Let $f=\sum a_{u} x^{u} \in \mathbb{C}\left[x_{1}^{ \pm 1}, \ldots, x_{n}^{ \pm 1}\right]$ and $V(f)$ the associated hypersurface in the algebraic torus $T^{n}=\left(\mathbb{C}^{*}\right)^{n}$. Then the tropical hypersurface of $f$ is

$$
\operatorname{trop}(V(f)):=\left\{w \in \mathbb{R}^{n} \begin{array}{l}
\text { the minimum in } f^{\operatorname{trop}}(w) \\
\text { is achieved at least twice }
\end{array}\right\} .
$$

Let $I$ be an ideal in $\mathbb{C}\left[x_{1}^{ \pm 1}, \ldots, x_{n}^{ \pm 1}\right]$. The tropicalization of the variety $V(I) \subset T^{n}$ is defined as

$$
\operatorname{trop}(V(I)):=\bigcap_{f \in I} \operatorname{trop}(V(f)) \subset \mathbb{R}^{n} .
$$

For a projective variety $V(I) \subset \mathbb{P}^{n-1}$ with $I$ a homogeneous ideal in $\mathbb{C}\left[x_{1}, \ldots, x_{n}\right]$ we consider the ideal $\hat{I}:=I \mathbb{C}\left[x_{1}^{ \pm 1}, \ldots, x_{n}^{ \pm 1}\right]$. Then $V(\hat{I})=V(I) \cap T^{n}$. The tropicalization of a projective variety is defined as $\operatorname{trop}(V(I)):=\operatorname{trop}(V(\hat{I}))$.

Recall the notion of initial ideal from Definiton 4 and consider for $w \in \mathbb{R}^{n}$ the initial ideal $\operatorname{in}_{w}(I)$. By [Eis95, Theorem 15.17], there exists a flat family over $\mathbb{C}$ whose generic fiber over $t \neq 0$ is isomorphic to $\mathbb{C}\left[x_{1}, \ldots, x_{n}\right] / I$ and whose special fiber over $t=0$ is isomorphic to $\mathbb{C}\left[x_{1}, \ldots, x_{n}\right] / \mathrm{in}_{w}(I)$. It is given by the following family of ideals

$$
\tilde{I}_{t}:=\left\langle t^{-\min _{u}\{w \cdot u\}} f\left(t^{w_{1}} x_{1}, \ldots, t^{w_{n}} x_{n}\right) \mid f=\sum a_{u} x^{u} \in I\right\rangle \subset \mathbb{C}\left[t, x_{1}^{ \pm 1}, \ldots, x_{n}^{ \pm 1}\right] .
$$

Let $I_{s}$ denote the ideal $\left.\tilde{I}_{t}\right|_{t=s}$. For $s \neq 0$ the isomorphism $\mathbb{C}\left[x_{1}, \ldots, x_{n}\right] / I_{s} \cong \mathbb{C}\left[x_{1}, \ldots, x_{n}\right] / I_{1}=$ $\mathbb{C}\left[x_{1}, \ldots, x_{n}\right]_{i} / I$ is given by a ring automorphism of $\mathbb{C}\left[x_{1}, \ldots, x_{n}\right]$ sending $I_{s}$ to $I$. If $\operatorname{in}_{w}(I)$ is toric, i.e. a binomial prime ideal, then $V\left(\mathrm{in}_{w}(I)\right)$ is a toric variety (see e.g. [MS15, Lemma 2.4.14]) and flat degeneration of $V(I)$ with family defined by $\tilde{I}_{t}$. To find toric initial ideals, it is reasonable to consider the tropicalization of $V(I)$ as due to the Fundamental Theorem of Tropical Geometry [MS15, Theorem 3.2.3] we have

$$
\operatorname{trop}(V(I))=\left\{w \in \mathbb{R}^{n} \mid \operatorname{in}_{w}(I) \text { is monomial-free }\right\} .
$$


Further, by the Structure Theorem [MS15, Theorem 3.3.5] trop $(V(I))$ is the support of a pure rational $d$-dimensional polyhedral fan, where $d$ is the Krull-dimension of $I$. It contains a linear subspace, called lineality space defined as

$$
\operatorname{trop}_{0}(V(I)):=\left\{w \in \operatorname{trop}(V(I)) \mid \operatorname{in}_{w}(I)=I\right\} .
$$

If $I$ is homogeneous we have $\mathbb{R}(1, \ldots, 1) \subset \operatorname{trop}_{0}(V(I))$. It is often convenient to consider the quotient of $\operatorname{trop}(V(I))$ by the lineality space. We can choose a fan structure on $\operatorname{trop}(V(I))$ by considering it as a subfan of the Gröbner fan of $I$. In particular, if $v$ and $w$ lie in the relative interior of a cone $C$, denoted by $v, w \in C^{\circ}$, if and only if

$$
\operatorname{in}_{w}(I)=\operatorname{in}_{v}(I) .
$$

We therefore adopt the notation $\operatorname{in}_{C}(I):=\operatorname{in}_{w}(I)$ for an arbitrary $w \in C^{\circ}$. We say a cone $C$ is prime, if $\operatorname{in}_{C}(I)$ is a prime ideal.

Definition 8. The tropical Grassmannian, denoted $\operatorname{trop}\left(\operatorname{Gr}\left(k, \mathbb{C}^{n}\right)\right) \subset \mathbb{R}^{\left(\begin{array}{l}n \\ k\end{array}\right)}$ is the tropical variety of the Plücker ideal $\mathcal{I}_{k, n}$. By [SS04, Corollary 3.1] it is a $k(n-k)+1$-dimensional polyhedral fan whose maximal cones are all of this dimension.

We mainly focus on the tropicalization of $\operatorname{Gr}\left(2, \mathbb{C}^{n}\right)$. By [SS04, Corollary 4.4] $\operatorname{trop}\left(\operatorname{Gr}\left(2, \mathbb{C}^{n}\right)\right)$ has the very nice property that every initial ideal $\operatorname{in}_{C}\left(\mathcal{I}_{2, n}\right)$ associated to a maximal cone $C \subset$ $\operatorname{trop}\left(\operatorname{Gr}\left(2, \mathbb{C}^{n}\right)\right)$ is toric. Further, Speyer and Sturmfels show the following:

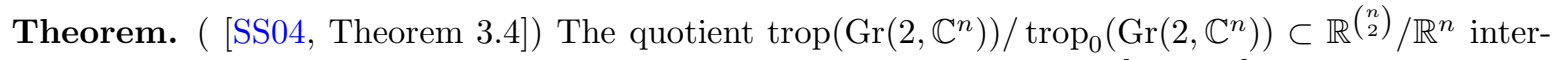
sected with the unit sphere is, up to sign, the space of phylogenetic trees [BHV01].

The theorem implies that every maximal prime cone $C$ can be associated with a labeled trivalent tree with $n$ leaves. The set of all labeled trivalent trees with $n$ leaves is denoted by $\mathcal{T}_{n}$. A trivalent tree is a graph with internal vertices of valency three and no loops or cycles of any kind. Non-internal vertices are called leaves and the word labeled refers to labeling the leaves by $1, \ldots, n$. We call an edge internal, if it connects two internal vertices.

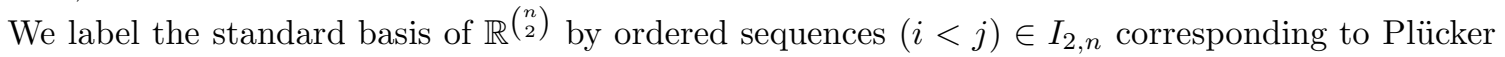
variables. The following definition shows how to obtain a point in the relative interior of a maximal cone in $\operatorname{trop}\left(\operatorname{Gr}\left(2, \mathbb{C}^{n}\right)\right)$ from a labeled trivalent tree. It follows from [SS04, Theorem 3.4].

Definition 9. Let $T$ be a labeled trivalent tree with $n$ leaves. Then the $(i<j)^{\prime}$ 'th entry of the weight vector $w_{T} \in \operatorname{trop}\left(\operatorname{Gr}\left(2, \mathbb{C}^{n}\right)\right)$ is

$$
\text { -\#\{internal edges on path from leaf } i \text { to leaf } j \text { in } T\} .
$$

For notational convenience we set $\operatorname{in}_{T}\left(\mathcal{I}_{2, n}\right):=\operatorname{in}_{\mathbf{w}_{T}}\left(\mathcal{I}_{2, n}\right)$. The corresponding maximal cone in $\operatorname{trop}\left(\operatorname{Gr}\left(2, \mathbb{C}^{n}\right)\right)$ is denoted $C_{T}$.

Remark 2. Combining the above, we have that every trivalent labeled tree induces a toric degeneration of $\operatorname{Gr}\left(2, \mathbb{C}^{n}\right)$ with flat family as given in (2.7). A main result of [KM19] is that there is a full-rank valuation with finitely generated value semi-group associated to prime cones in the tropicalization of a projective variety. This yields a flat family using Rees algebras as in Definition 2.

The symmetric group $S_{n}$ acts on $\mathcal{T}_{n}$ by permuting the labels of the leaves of a tree. We also have an $S_{n}$-action on Plücker coordinates given by

$$
\sigma\left(p_{i j}\right)=\operatorname{sgn}(\sigma) p_{\sigma^{-1}(i) \sigma^{-1}(j)} \text { for } \sigma \in S_{n} .
$$

If $\sigma^{-1}(i)>\sigma^{-1}(j)$, we set $p_{\sigma^{-1}(i) \sigma^{-1}(j)}:=-p_{\sigma^{-1}(j) \sigma^{-1}(i)}$. The $S_{n}$-action induces a ring automorphism of $\mathbb{C}\left[p_{i j}\right]_{i<j}$ for every $\sigma \in S_{n}$. It sends in $\operatorname{in}_{T}\left(\mathcal{I}_{2, n}\right)$ to $\operatorname{in}_{\sigma(T)}\left(\mathcal{I}_{2, n}\right)$ for every trivalent labeled tree $T \in \mathcal{T}_{n}$. Denote the equivalence class of $T$ with respect to this action by $\mathrm{T}$. It is uniquely determined by the 


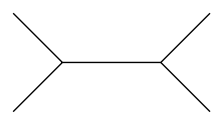

Figure 2: A trivalent tree with 4 leaves.

underlying (unlabeled) trivalent tree with $n$ leaves, see for example Figure 2. We denote the set of trivalent trees by $\mathcal{T}_{n} / S_{n}$.

Consider a trivalent tree $\mathrm{T} \in \mathcal{T}_{n} / S_{n}$. If there are two non-internal edges connected to the same internal vertex $c$, then we say $\mathrm{T}$ has a cherry at vertex $c$.

Lemma 1. Every trivalent tree with $n \geq 4$ leaves has a cherry.

Proof. We use induction on $n$. For $n=4$ Figure 2 displays the only trivalent tree in $\mathcal{T}_{4} / S_{4}$ and we see, it has two cherries. Now consider a trivalent tree $\mathrm{T}^{\prime} \in \mathcal{T}_{n+1} / S_{n+1}$. We remove one edge connected to a leaf and obtain a tree $\mathrm{T} \in \mathcal{T}_{n} / S_{n}$. By induction, $\mathrm{T}$ has a cherry at some vertex $c$. Adding the removed edge back there are two possibilities: either we add it to an internal edge (creating a new internal vertex), then the cherry also exists in $\mathrm{T}^{\prime}$. Or we add it at an edge with a leaf, hence create a new cherry.

\section{Birational sequences}

We recall the definition of birational sequences due to Fang, Fourier, and Littelmann in [FFL17] and associated valuations. After proving the central Lemma 2 we define a new class of birational sequences called iterated in Definition 11.

From now on let $d:=k(n-k)$ be the dimension of $\operatorname{Gr}\left(k, \mathbb{C}^{n}\right)$. Consider a positive root $\beta \in R^{+}$. The root subgroup corresponding to $\beta$ is given by $U_{-\beta}:=\left\{\exp \left(z f_{\beta}\right) \mid z \in \mathbb{C}\right\} \subset U^{-}$, where $\exp \left(z f_{\beta}\right)=$ $\mathbb{1}+z f_{\beta}$.

Definition 10. ( [FFL17, Definition 2]) Let $S=\left(\beta_{1}, \ldots, \beta_{d}\right)$ be a sequence of positive roots. Then $S$ is called a birational sequence for $\operatorname{Gr}\left(k, \mathbb{C}^{n}\right)$ if the multiplication map

$$
\psi_{S}:=\text { mult }: U_{-\beta_{1}} \times \cdots \times U_{-\beta_{d}} \rightarrow U^{-}
$$

has image birational to $U_{k}^{-}$.

Notice that $U_{-\beta_{1}} \times \cdots \times U_{-\beta_{d}} \cong \mathbb{A}^{d}$.

Example 2. The following are two first (and motivating) examples of birational sequences:

1. The multiplication map $\prod_{\beta \in R_{k}^{+}} U_{-\beta} \rightarrow U^{-}$has image $U_{k}^{-}$and hence, is birational. In particular, every sequence containing all roots in $R_{k}^{+}$(in arbitrary order) is a birational sequence called $P B W$-sequence, see [FFL17, Example 1 and page 131]. We distinguish between PBW-sequences $S$ and $S^{\prime}$ when the order of the roots in $S$ is different from the order of the roots in $S^{\prime}$.

2. For $w_{0}$ the longest element in $S_{n}$, let $w_{k} \in S_{n} /\left\langle s_{i} \mid i \neq k\right\rangle$ denote a coset representative of $w_{0}$. Fix a reduced decomposition $\underline{w}_{k}=s_{i_{1}} \ldots s_{i_{d}}$ of $w_{k}$ and let $S=\left(\varepsilon_{i_{1}}-\varepsilon_{i_{1}+1}, \ldots, \varepsilon_{i_{d}}-\varepsilon_{i_{d}+1}\right)$ be the corresponding sequence of simple roots. Then $S$ is a birational sequence, it is referred to as the reduced decomposition case in [FFL17, Example 2].

The second example shows that repetitions of positive roots may occur in birational sequences. Our aim is to shed some light on sequences that are neither PBW nor associated to reduced decompositions for Grassmannians. The following lemma allows us to construct such sequences for $\operatorname{Gr}\left(k, \mathbb{C}^{n+1}\right)$ from sequences for $\operatorname{Gr}\left(k, \mathbb{C}^{n}\right)$. 
Lemma 2. Let $S=\left(\beta_{1}, \ldots, \beta_{d}\right)$ be a birational sequence for $\operatorname{Gr}\left(k, \mathbb{C}^{n}\right)$ and chose $I=\left\{i_{1}, \ldots, i_{k}\right\} \subset[n]$ with $|I|=k$. Then the following is a birational sequence for $\operatorname{Gr}\left(k, \mathbb{C}^{n+1}\right)$ :

$$
S^{\prime}:=\left(\varepsilon_{i_{1}}-\varepsilon_{n+1}, \ldots, \varepsilon_{i_{k}}-\varepsilon_{n+1}, \beta_{1}, \ldots, \beta_{d}\right) .
$$

Proof. The elements of $\operatorname{im}\left(\psi_{S^{\prime}}\right)$ are of the form

$$
\exp \left(y_{1} f_{i_{1}, n+1}\right) \cdots \exp \left(y_{k} f_{i_{k}, n+1}\right) \exp \left(z_{1} f_{\beta_{1}}\right) \cdots \exp \left(z_{d} f_{\beta_{d}}\right)=\left(\begin{array}{cccc}
1 & 0 & \cdots & 0 \\
a_{2,1} & 1 & \ldots & 0 \\
\vdots & \ddots & \ddots & 0 \\
a_{n+1,1} & \ldots & a_{n+1, n} & 1
\end{array}\right),
$$

for $y_{1}, \ldots, y_{k}, z_{1}, \ldots, z_{d} \in \mathbb{C}$. They satisfy $a_{n+1, j}=y_{1} a_{i_{1}, j}+\cdots+y_{k} a_{i_{k}, j}$ for $1 \leq i \leq n$. Deleting the last row and column gives the image of $\psi_{S}$, i.e. for $1 \leq i<j \leq n$ we have $a_{i, j} \in \mathbb{C}\left[z_{1}, \ldots, z_{d}\right]$. We need to show that $\operatorname{im}\left(\psi_{S^{\prime}}\right)$ is birational to $U_{k}^{-} \subset S L_{n+1}$ given that $\operatorname{im}\left(\psi_{S}\right)$ is birational to $U_{k}^{-} \subset S L_{n}$. Denote the corresponding map for $S$ by $\phi_{S}: \operatorname{im}\left(\psi_{S}\right) \rightarrow U_{k}^{-} \subset S L_{n}$. Recall that elements of $U_{k}^{-}$are block matrices of form

$$
\left(x_{i, j}\right)_{i, j}=\left(\begin{array}{ll}
\mathbb{1} & 0 \\
* & \mathbb{1}
\end{array}\right)
$$

where non-trivial $x_{i, j}$ occur for $1 \leq j \leq k$ and $k+1 \leq i \leq n$. Let $\underline{i} \in I_{k, n}$ be the orderd sequence with entries $i_{1}, \ldots, i_{k}$. We define a birational map $\phi_{S^{\prime}}: \operatorname{im}\left(\psi_{S^{\prime}}\right) \rightarrow U^{-} \subset S L_{n+1}$ by specifying for $1 \leq j \leq k$

$$
y_{j} \mapsto \frac{(-1)^{k-j+1} \bar{p}_{\underline{i} \backslash i_{j} \cup n+1}}{\bar{p}_{\underline{i}}} \quad \text { and } \quad a_{r, s} \mapsto \phi_{S}\left(a_{r, s}\right)=x_{r, s} \quad \text { if } \quad 1 \leq r, s \leq n .
$$

Then $\phi_{S^{\prime}}\left(a_{n+1, j}\right)=\sum_{l=1}^{k} \phi_{S^{\prime}}\left(y_{l}\right) \phi_{S}\left(a_{i_{l}, j}\right)$. Before continuing with the computation note that on $U_{k}^{-} \subset S L_{n+1}$ we have $\bar{p}_{(1<\cdots<k) \backslash j \cup i_{l}}\left(\left(x_{r, s}\right)_{r, s}\right)=x_{i_{l}, j}$ for $1 \leq l \leq k$. If $j \leq k$, making use of the Plücker relations (2.1) this yields the following:

$$
\begin{aligned}
\phi_{S^{\prime}}\left(a_{n+1, j}\right) & =\sum_{l=1}^{k} \phi_{S^{\prime}}\left(y_{l}\right) \phi_{S}\left(a_{i_{l}, j}\right)=\sum_{l=1}^{k} \frac{(-1)^{k-l+1} \bar{p}_{\underline{i} \backslash i_{l} \cup n+1}}{\bar{p}_{\underline{i}}} x_{i_{l}, j} \\
& =\sum_{l=1}^{k} \frac{(-1)^{k-l+1} \bar{p}_{\underline{i} \backslash i_{l} \cup n+1}}{\bar{p}_{\underline{i}}} \bar{p}_{(1<\cdots<k) \backslash j \cup i_{l}}=\bar{p}_{(1<\cdots<k) \backslash j \cup n+1}=x_{n+1, j} .
\end{aligned}
$$

For $l>k$ we obtain $\phi_{S^{\prime}}\left(a_{n+1, l}\right) \in \mathbb{C}\left(x_{i, j} \mid 1 \leq j \leq k, k+1 \leq i \leq n\right)$. In particular, we have $\mathbb{C}\left(\operatorname{im}\left(\phi_{S^{\prime}}\right)\right) \cong$ $\mathbb{C}\left(U_{k}^{-}\right) \cong \mathbb{C}\left(\operatorname{Gr}\left(k, \mathbb{C}^{n+1}\right)\right)$. By little abuse of notation we denote the invertible maps induced by $S$ between $\mathbb{C}\left(\operatorname{Gr}\left(k, \mathbb{C}^{n}\right)\right)$ and $\mathbb{C}\left(\mathbb{A}^{d}\right) \cong \mathbb{C}\left(z_{1}, \ldots, z_{d}\right)$ by $\psi_{S}^{*}$ and $\left(\psi_{S}^{*}\right)^{-1}$, respectively. Induced by the pullback of $\psi_{S^{\prime}}$ we consider the map between the rings of rational functions (also denoted $\psi_{S^{\prime}}^{*}$ ):

$$
\begin{aligned}
\psi_{S^{\prime}}^{*}: \mathbb{C}\left(\operatorname{Gr}\left(k, \mathbb{C}^{n+1}\right)\right) & \rightarrow \mathbb{C}\left(\mathbb{A}^{d+k}\right) \cong \mathbb{C}\left(z_{1}, \ldots, z_{d}, y_{1}, \ldots, y_{k}\right) \\
\bar{p}_{\underline{j}} & \mapsto\left\{\begin{array}{ccc}
\psi_{S}^{*}\left(\bar{p}_{j}\right) & \text { if } \quad \underline{j} \in I_{k, n}, \\
\sum_{l=1}^{k} y_{l} \psi_{S}^{*}\left((-1)^{\ell\left(\underline{\hat{j}}, i_{l}\right)} \bar{p}_{\left.\underline{j} \backslash n+1 \cup i_{l}\right)}\right. & \text { if } & n+1 \in \underline{j} .
\end{array}\right.
\end{aligned}
$$

A straightforward computation reveals that the following map is indeed inverse to $\psi_{S^{\prime}}^{*}$ above

$$
\begin{aligned}
\left(\psi_{S^{\prime}}^{*}\right)^{-1}: \mathbb{C}\left(\mathbb{A}^{d+k}\right) & \rightarrow \mathbb{C}\left(\operatorname{Gr}\left(k, \mathbb{C}^{n+1}\right)\right) \cong \mathbb{C}\left(\bar{p}_{\underline{j}}\right)_{\underline{j} \in I_{k, n+1}} \\
z_{i} & \mapsto\left(\psi_{S}^{*}\right)^{-1}\left(z_{i}\right) \in \mathbb{C}\left(\bar{p}_{\underline{j}}\right)_{\underline{j} \in I_{k, n}}, \\
y_{j} & \mapsto \frac{(-1)^{k-j+1} \bar{p}_{\underline{i} \backslash i_{j} \cup n+1}}{\bar{p}_{\underline{i}}}
\end{aligned}
$$

which completes the proof. 
Definition 11. For $k<n$ consider a birational sequence for $\operatorname{Gr}\left(k, \mathbb{C}^{k+1}\right)$. Now extend it as in (3.1) to a birational sequence for $\operatorname{Gr}\left(k, \mathbb{C}^{k+2}\right)$. Repeat this process until the outcome is a birational sequence for $\operatorname{Gr}\left(k, \mathbb{C}^{n}\right)$. Birational sequences of this form are called iterated.

We explain how to obtain a valuation from a fixed birational sequence $S=\left(\beta_{1}, \ldots, \beta_{d}\right)$ for $\operatorname{Gr}\left(k, \mathbb{C}^{n}\right)$ as constructed in [FFL17, §7]. Define the height function ht: $R^{+} \rightarrow \mathbb{Z}_{>0}$ by ht $\left(\varepsilon_{i}-\varepsilon_{j}\right):=j-i$. Then the height weighted function $\Psi_{S}: \mathbb{Z}^{d} \rightarrow \mathbb{Z}$ is given by

$$
\Psi_{S}\left(m_{1}, \ldots, m_{d}\right):=\sum_{i=1}^{d} m_{i} \operatorname{ht}\left(\beta_{i}\right) .
$$

Let $<_{\text {lex }}$ be the lexicographic order on $\mathbb{Z}^{d}$. The $\Psi_{S}$-weighted reverse lexicographic order $\prec_{\Psi_{S}}$ on $\mathbb{Z}^{d}$ is defined by setting for $m, n \in \mathbb{Z}^{d}$

$$
m \prec_{\Psi_{S}} n: \Leftrightarrow \Psi_{S}(m)<\Psi_{S}(n) \text { or } \Psi_{S}(m)=\Psi_{S}(n) \text { and } m>_{\text {lex }} n .
$$

When the sequence $S$ is clear from the context we denote $\Psi_{S}$ by $\Psi$.

Definition 12. ( $[$ FFL17, $\S 7])$ Let $f=\sum a_{u} y^{u}$ with $u \in \mathbb{Z}_{\geq 0}^{d}$ be a non-zero polynomial in $\mathbb{C}\left[y_{1}, \ldots, y_{d}\right]$. The valuation $\mathfrak{v}_{S}: \mathbb{C}\left[y_{1}, \ldots, y_{d}\right] \backslash\{0\} \rightarrow\left(\mathbb{Z}_{\geq 0}^{d}, \prec \Psi\right)$ associated to $S$ is defined as

$$
\mathfrak{v}_{S}(f):=\min \prec_{\Psi}\left\{u \in \mathbb{Z}_{\geq 0}^{d} \mid a_{u} \neq 0\right\} .
$$

We extend $\mathfrak{v}_{S}$ to a valuation on $\mathbb{C}\left(y_{1}, \ldots, y_{d}\right) \backslash\{0\}$ by $\mathfrak{v}_{S}\left(\frac{f}{g}\right):=\mathfrak{v}_{S}(f)-\mathfrak{v}_{S}(g)$ for $f, g \in \mathbb{C}\left[y_{1}, \ldots, y_{d}\right] \backslash\{0\}$.

Valuations of form (3.3) are called lowest term valuations. As $S$ is a birational sequence, for every $f \in \mathbb{C}\left(\operatorname{Gr}\left(k, \mathbb{C}^{n}\right)\right)$ there exists a unique element $\psi_{S}^{*}(f) \in \mathbb{C}\left(y_{1}, \ldots, y_{d}\right)$. Hence, we have a valuation on $\mathbb{C}\left(\operatorname{Gr}\left(k, \mathbb{C}^{n}\right)\right) \backslash\{0\}$ given by $\mathfrak{v}_{S}(f):=\mathfrak{v}_{S}\left(\psi_{S}^{*}(f)\right)$. We restrict to obtain

$$
\mathfrak{v}_{S}: A_{k, n} \backslash\{0\} \rightarrow\left(\mathbb{Z}_{\geq 0}^{d}, \prec \Psi\right) .
$$

Denote by $S\left(A_{k, n}, \mathfrak{v}_{S}\right)$ the associated value semi-group and the associated graded algebra by $\operatorname{gr}_{S}\left(A_{k, n}\right)$. For the images of Plücker coordinates $\bar{p}_{\underline{j}} \in A_{k, n}$ we choose as before the notation $\overline{p_{\underline{j}}} \in \operatorname{gr}_{S}\left(A_{k, n}\right)$. The weighting matrix for $\mathfrak{v}_{S}$ is denoted $M_{S}$.

Theorem 1. Let $S$ be an iterated sequence for $\operatorname{Gr}\left(k, \mathbb{C}^{n}\right)$ and $\mathfrak{v}_{S}: A_{k, n} \backslash\{0\} \rightarrow\left(\mathbb{Z}_{\geq 0}^{d}, \prec \Psi\right)$ the corresponding valuation. Then there exists a cone $C \subset \operatorname{trop}\left(\operatorname{Gr}\left(k, \mathbb{C}^{n}\right)\right)$ such that

$$
\operatorname{in}_{M_{S}}\left(\mathcal{I}_{k, n}\right)=\operatorname{in}_{C}\left(\mathcal{I}_{k, n}\right) .
$$

Proof. We need to show that $\mathfrak{v}_{S}$ is a full rank valuation. Then by [Bos20, Corollary 3] the initial ideal $\operatorname{in}_{M_{\mathfrak{v}}}\left(\mathcal{I}_{k, n}\right)$ is monomial-free. Moreover, there exists $w \in \mathbb{Z}^{\left(\begin{array}{l}n \\ k\end{array}\right)}$ with $\operatorname{in}_{w}\left(\mathcal{I}_{k, n}\right)=\operatorname{in}_{M_{\mathfrak{v}}}\left(\mathcal{I}_{k, n}\right)$. In particular, we have a cone $C \subset \operatorname{trop}\left(\operatorname{Gr}\left(k, \mathbb{C}^{n}\right)\right)$ with $w \in C^{\circ}$.

To prove that $\mathfrak{v}_{S}$ has full rank, it suffices to show that $M_{S}$ has full rank. As $S$ is an iterated sequence we pursue by induction on $n$. If $n=k+1$ we may assume that $S=\left(\varepsilon_{1}-\varepsilon_{k+1}, \ldots, \varepsilon_{k}-\varepsilon_{k+1}\right)$. In this case, after choosing an apropriate order on Plücker coordinates, $M_{S}$ contains the identity matrix as a submatrix. Now assume that the claim is true for $n-1$ and let $S=\left(\varepsilon_{i_{1}}-\varepsilon_{n}, \ldots, \varepsilon_{i_{k}}-\varepsilon_{n}, \beta_{1}, \ldots, \beta_{d^{\prime}}\right)$, where $\left(\beta_{1}, \ldots, \beta_{d^{\prime}}\right)$ is an iterated sequence for $\operatorname{Gr}\left(k, \mathbb{C}^{n-1}\right)$. We have to show that the rows of $M_{S}$ corresponding to $\varepsilon_{i_{1}}-\varepsilon_{n}, \ldots, \varepsilon_{i_{k}}-\varepsilon_{n}$ are linearly independent. Consider $\underline{j}^{1}, \ldots, \underline{j}^{k} \in I_{k, n}$ such that for all $l \leq k$ we have $i_{1}, \ldots, i_{l-1}, n \in \underline{j}^{l}$ and $i_{l} \notin \underline{j}^{l}$. Compute the columns of $M_{S}$ corresponding to $\mathfrak{v}_{S}\left(\bar{p}_{\underline{j}^{1}}\right), \ldots, \mathfrak{v}_{S}\left(\bar{p}_{\underline{j}^{k}}\right)$ using [FFL17, Proposition 2]. We see that the submatrix with rows corresponding to $\varepsilon_{i_{1}}-\varepsilon_{n}, \ldots, \bar{\varepsilon}_{i_{k}}-\varepsilon_{n}$ is a $k \times k$-identity matrix. Hence, the columns of $\mathfrak{v}_{S}\left(\bar{p}_{\underline{j}^{1}}\right), \ldots, \mathfrak{v}_{S}\left(\bar{p}_{\underline{j}}\right)$ are linearly independent and so $M_{S}$ is of full rank by induction.

Corollary 1. For every iterated sequence $S$ the weighting matrix $M_{S}$ is of full rank. 


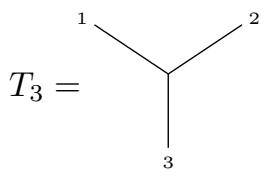

Figure 3: labeled trivalent tree with three leaves.

\section{Iterated sequences for $\operatorname{Gr}\left(2, \mathbb{C}^{n}\right)$}

In this subsection we prove Theorem 2 stated in the introduction. After proving Proposition 1 we can apply [Bos20, Theorem 1] to complete the proof. We focus on iterated sequences for $\operatorname{Gr}\left(2, \mathbb{C}^{n}\right)$ and start by computing the images of Plücker coordinates under the associated valuations.

Let $S=\left(\beta_{1}, \ldots, \beta_{d}\right)$ be a birational sequence for $\operatorname{Gr}\left(2, \mathbb{C}^{n}\right)$. Let $U\left(\mathfrak{n}_{S}^{-}\right) \subset U\left(\mathfrak{n}^{-}\right)$be the subalgebra generated by monomials of form $f_{\beta_{1}}^{m_{1}} \ldots f_{\beta_{d}}^{m_{d}}$. We consider the irreducible highest weight representation $V\left(\omega_{2}\right)=\bigwedge^{2} \mathbb{C}^{n}=U\left(\mathfrak{n}_{S}^{-}\right) \cdot\left(e_{1} \wedge e_{2}\right)$. There exists at least one monomial of form $\mathbf{f}_{S}^{m}=f_{\beta_{1}}^{m_{1}} \ldots f_{\beta_{d}}^{m_{d}}$ with the property $\mathbf{f}_{S}^{m} \cdot\left(e_{1} \wedge e_{2}\right)=e_{i} \wedge e_{j}$ for all $1 \leq i, j \leq n$. Then by [FFL17, Proposition 2] we have

$$
\mathfrak{v}_{S}\left(\bar{p}_{i j}\right)=\min _{\prec_{\Psi}}\left\{m \in \mathbb{Z}_{\geq 0}^{d} \mid \mathbf{f}_{S}^{m} \cdot\left(e_{1} \wedge e_{2}\right)=e_{i} \wedge e_{j}\right\} .
$$

Example 3. Consider $\operatorname{Gr}\left(2, \mathbb{C}^{4}\right)$ and the iterated sequence $S=\left(\varepsilon_{1}-\varepsilon_{4}, \varepsilon_{2}-\varepsilon_{4}, \varepsilon_{1}-\varepsilon_{3}, \varepsilon_{2}-\varepsilon_{3}\right)$, respectively $S^{\prime}=\left(\varepsilon_{3}-\varepsilon_{4}, \varepsilon_{2}-\varepsilon_{4}, \varepsilon_{1}-\varepsilon_{3}, \varepsilon_{2}-\varepsilon_{3}\right)$. They are birational by Lemma 2 , as $\left(\varepsilon_{1}-\varepsilon_{3}, \varepsilon_{2}-\varepsilon_{3}\right)$ is of PBW-type for $\operatorname{Gr}\left(2, \mathbb{C}^{3}\right)$. We compute the valuation $\mathfrak{v}_{S}$ on Plücker coordinates. There are two monomials sending $e_{1} \wedge e_{2}$ to $e_{3} \wedge e_{4}$, namely

$$
\mathbf{f}_{S}^{(1,0,0,1)} \cdot\left(e_{1} \wedge e_{2}\right)=\mathbf{f}_{S}^{(0,1,1,0)} \cdot\left(e_{1} \wedge e_{2}\right)=e_{1} \wedge e_{4} .
$$

We have $\Psi_{S}(1,0,0,1)=\Psi_{S}(0,1,1,0)=4$, but $(1,0,0,1)>_{\text {lex }}(0,1,1,0)$. Hence, $\mathfrak{v}_{S}\left(\bar{p}_{34}\right)=(1,0,0,1)$.

For $\mathfrak{v}_{S^{\prime}}$ we compute $\mathbf{f}_{S}^{(1,0,0,1)}\left(e_{1} \wedge e_{2}\right)=\mathbf{f}_{S}^{(0,1,0,0)}\left(e_{1} \wedge e_{2}\right)=e_{1} \wedge e_{4}$ Again, we have $\Psi_{S^{\prime}}(1,0,0,1)=$ $\Psi_{S^{\prime}}(0,1,0,0)=2$, but as $(1,0,0,1)>_{\text {lex }}(0,1,0,0)$ it follows $\mathfrak{v}_{S^{\prime}}\left(\bar{p}_{14}\right)=(1,0,0,1)$. In Table 1 you can find the images of all Plücker coordinates under $\mathfrak{v}_{S}$ and $\mathfrak{v}_{S^{\prime}}$.

\begin{tabular}{|c|c|c|}
\hline Plücker & $\mathfrak{v}_{S}$ & $\mathfrak{v}_{S^{\prime}}$ \\
\hline $\bar{p}_{12}$ & $(0,0,0,0)$ & $(0,0,0,0)$ \\
$\bar{p}_{13}$ & $(0,0,0,1)$ & $(0,0,0,1)$ \\
$\bar{p}_{23}$ & $(0,0,1,0)$ & $(0,0,1,0)$ \\
$\bar{p}_{14}$ & $(0,1,0,0)$ & $(1,0,0,1)$ \\
$\bar{p}_{24}$ & $(1,0,0,0)$ & $(1,0,1,0)$ \\
$\bar{p}_{34}$ & $(1,0,0,1)$ & $(0,1,1,0)$ \\
\hline
\end{tabular}

Table 1: Images of Plücker coordinates under the valuations $\mathfrak{v}_{S}, \mathfrak{v}_{S^{\prime}}$ associated to $S=\left(\varepsilon_{1}-\varepsilon_{4}, \varepsilon_{2}-\right.$ $\left.\varepsilon_{4}, \varepsilon_{1}-\varepsilon_{3}, \varepsilon_{2}-\varepsilon_{3}\right)$, respectively $S^{\prime}=\left(\varepsilon_{3}-\varepsilon_{4}, \varepsilon_{2}-\varepsilon_{4}, \varepsilon_{1}-\varepsilon_{3}, \varepsilon_{2}-\varepsilon_{3}\right)$ for $\operatorname{Gr}\left(2, \mathbb{C}^{4}\right)$.

From now on we fix an iterated birational sequence $S=\left(\varepsilon_{i_{n}}-\varepsilon_{n}, \varepsilon_{j_{n}}-\varepsilon_{n}, \ldots, \varepsilon_{i_{3}}-\varepsilon_{3}, \varepsilon_{j_{3}}-\varepsilon_{3}\right)$ for $\operatorname{Gr}\left(2, \mathbb{C}^{n}\right)$, where $i_{l}, j_{l} \in[l-1]$ with $i_{l} \neq j_{l}$ for all $3 \leq l \leq n$. Then Algorithm 1 associates to $S$ a labeled trivalent tree $T_{S}$ with $n$ leaves.

Definition 13. To an iterated sequence $S$ we associate the trivalent tree $T_{S}$ and the sequence of trees $\mathbb{T}_{S}=\left(T_{n}^{S}, \ldots, T_{3}^{S}\right)$ that are the output of Algorithm 1. Denote by $C_{S}$ the maximal cone in $\operatorname{trop}\left(\operatorname{Gr}\left(2, \mathbb{C}^{n}\right)\right)$ corresponding to the tree $T_{S}$ by [SS04, Theorem 3.4]. 


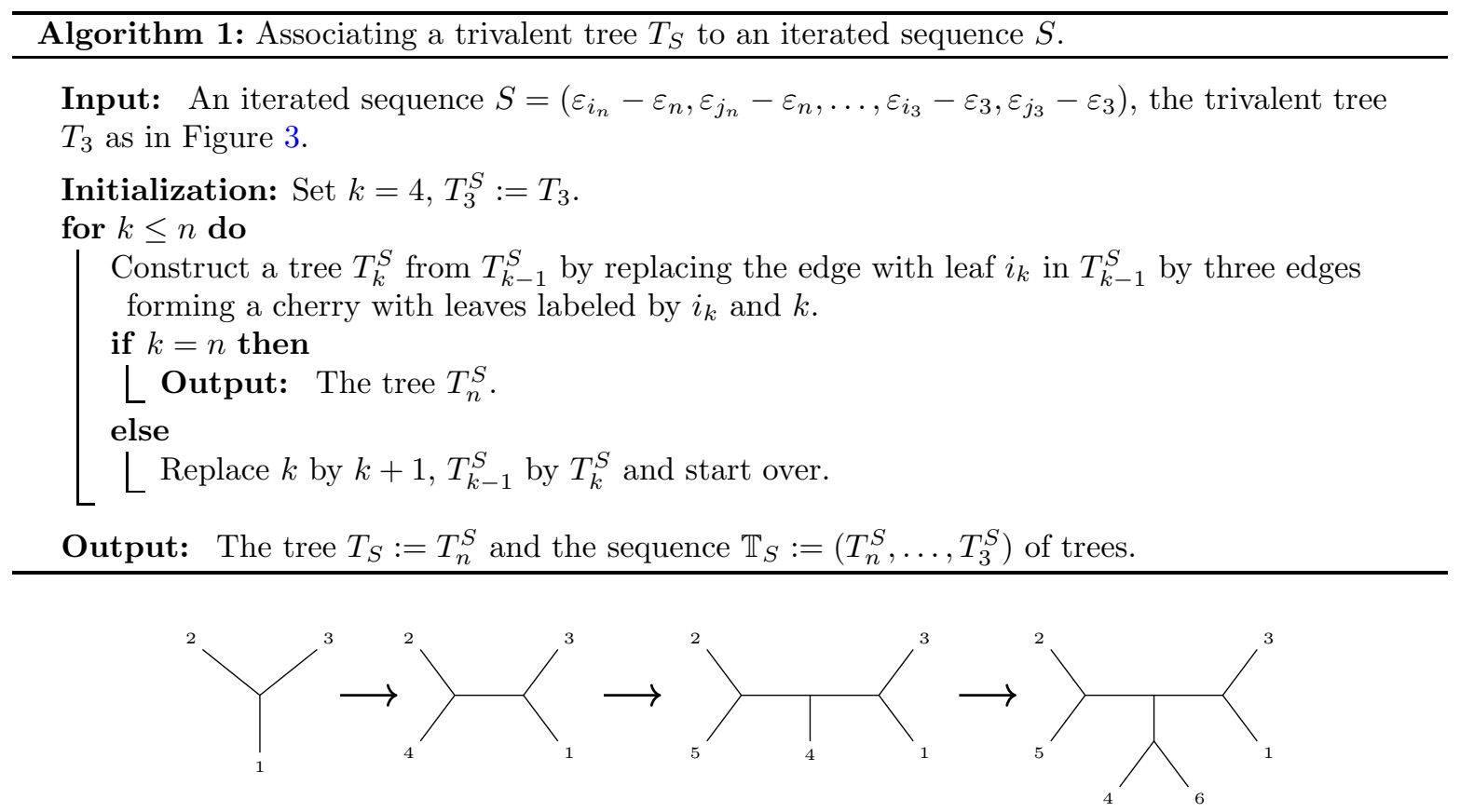

Figure 4: The sequence $\mathbb{T}_{S}$ for $S$ as in Example 4.

Example 4. Consider $S=\left(\varepsilon_{4}-\varepsilon_{6}, \varepsilon_{5}-\varepsilon_{6}, \varepsilon_{2}-\varepsilon_{5}, \varepsilon_{3}-\varepsilon_{5}, \varepsilon_{2}-\varepsilon_{4}, \varepsilon_{3}-\varepsilon_{4}, \varepsilon_{1}-\varepsilon_{3}, \varepsilon_{2}-\varepsilon_{3}\right)$, an iterated sequence for $\operatorname{Gr}\left(2, \mathbb{C}^{6}\right)$. We construct the trees $\mathbb{T}_{S}=\left(T_{3}^{S}, T_{4}^{S}, T_{5}^{S}, T_{6}^{S}\right)$ by Algorithm 1 . Figure 4 shows the obtained sequence of trees.

Let $M_{S}:=M_{\mathfrak{v}_{S}}$ be the weighting matrix associated to $\mathfrak{v}_{S}$ as in Definition 5 . We compute the initial ideal $\operatorname{in}_{M_{S}}\left(\mathcal{I}_{2, n}\right)$ of the Plücker ideal $\mathcal{I}_{2, n}$ to apply [Bos20, Theorem 1].

Proposition 1. For every iterated sequence $S$ we have $\operatorname{in}_{M_{S}}\left(\mathcal{I}_{2, n}\right)=\operatorname{in}_{C_{S}}\left(\mathcal{I}_{2, n}\right)$.

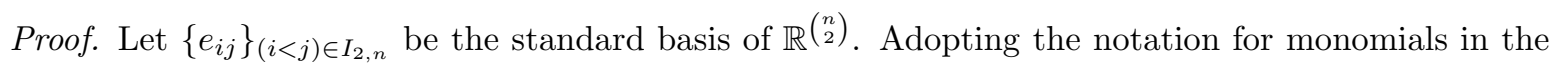
polynomial ring $\mathbb{C}\left[p_{i j}\right]_{i<j}$ we have

$$
R_{i, j, k, l}=p_{i j} p_{k l}-p_{i k} p_{j l}+p_{i l} p_{j k}=\mathbf{p}^{e_{i j}+e_{k l}}-\mathbf{p}^{e_{i k}+e_{j l}}+\mathbf{p}^{e_{i l}+e_{j k}}
$$

In particular, $M_{S}\left(e_{i j}+e_{k l}\right)=\mathfrak{v}_{S}\left(\bar{p}_{i j}\right)+\mathfrak{v}_{S}\left(\bar{p}_{k l}\right)=\mathfrak{v}_{S}\left(\bar{p}_{i j} \bar{p}_{k l}\right)$ and $\operatorname{in}_{M_{S}}\left(R_{i, j, k, l}\right)$ is the sum of those monomials in $R_{i, j, k, l}$ for which the valuation $\mathfrak{v}_{S}$ of the corresponding monomials in $A_{2, n}$ is minimal with respect to $\prec_{\Psi}$.

Recall that $\operatorname{in}_{C_{S}}\left(\mathcal{I}_{2, n}\right)=\left\langle\operatorname{in}_{C_{S}}\left(R_{i, j, k, l}\right) \mid 1 \leq i<j<k<l \leq n\right\rangle$ by [SS04, Proof of Theorem 3.4]. This implies that it is enough to prove the following claim.

Claim: For every Plücker relation $R_{i, j, k, l}$ with $1 \leq i<j<k<l \leq n$ we have $\operatorname{in}_{C_{S}}\left(R_{i, j, k, l}\right)=$ $\operatorname{in}_{M_{S}}\left(R_{i, j, k, l}\right)$.

Proof of claim: We proceed by induction. For $n=4$ let $S=\left(\varepsilon_{i}-\varepsilon_{4}, \varepsilon_{j}-\varepsilon_{4}, \varepsilon_{i_{3}}-\varepsilon_{3}, \varepsilon_{j_{3}}-\varepsilon_{3}\right)$, i.e. the

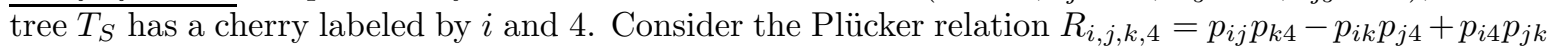
with $\{i, j, k\}=[3]$. Then

$$
\operatorname{in}_{C_{S}}\left(R_{i, j, k, 4}\right)=p_{i j} p_{k 4}-p_{i k} p_{j 4} \text {. }
$$

Let $S^{\prime}=\left(\varepsilon_{i_{3}}-\varepsilon_{3}, \varepsilon_{j_{3}}-\varepsilon_{3}\right)$ be the sequence for $\operatorname{Gr}\left(2, \mathbb{C}^{3}\right)$ and denote by $\hat{p}_{r s}$ with $(r<s) \in$ $I_{2,3}$ the Plücker coordinates in $A_{2,3}$. For $m=\left(m_{d-2}, \ldots, m_{1}\right) \in \mathbb{Z}^{d-2}$ and $m_{d}, m_{d-1} \in \mathbb{Z}$ write 
$\left(m_{d}, m_{d-1}, m\right):=\left(m_{d}, m_{d-1}, m_{d-2}, \ldots, m_{1}\right)$ We compute

$$
\mathfrak{v}_{S}\left(\bar{p}_{i 4}\right)=\left(0,1, \mathfrak{v}_{S^{\prime}}\left(\hat{p}_{i j}\right)\right), \quad \mathfrak{v}_{S}\left(\bar{p}_{j 4}\right)=\left(1,0, \mathfrak{v}_{S^{\prime}}\left(\hat{p}_{i j}\right)\right), \quad \text { and } \mathfrak{v}_{S}\left(\bar{p}_{k 4}\right)=\left(1,0, \mathfrak{v}_{S^{\prime}}\left(\hat{p}_{i k}\right)\right) .
$$

This implies $\mathfrak{v}_{S}\left(\bar{p}_{i 4} \bar{p}_{j k}\right) \succ_{\Psi} \mathfrak{v}_{S}\left(\bar{p}_{i j} \bar{p}_{k 4}\right)=\mathfrak{v}_{S}\left(\bar{p}_{i k} \bar{p}_{j 4}\right)$, and hence $\operatorname{in}_{M_{S}}\left(R_{i, j, k, 4}\right)=\operatorname{in}_{C_{S}}\left(R_{i, j, k, 4}\right)$.

Assume the claim is true for $n-1$ and let $S=\left(\varepsilon_{i_{n}}-\varepsilon_{n}, \varepsilon_{j_{n}}-\varepsilon_{n}, \ldots, \varepsilon_{i_{3}}-\varepsilon_{3}, \varepsilon_{j_{3}}-\varepsilon_{3}\right)$ be an iterated sequence for $\operatorname{Gr}\left(2, \mathbb{C}^{n}\right)$. Then $S^{\prime}=\left(\varepsilon_{i_{n-1}}-\varepsilon_{n-1}, \varepsilon_{j_{n-1}}-\varepsilon_{n-1}, \ldots, \varepsilon_{i_{3}}-\varepsilon_{3}, \varepsilon_{j_{3}}-\varepsilon_{3}\right)$ is an iterated sequence for $\operatorname{Gr}\left(2, \mathbb{C}^{n-1}\right)$. Denote by $\hat{p}_{i j}$ with $(i<j) \in I_{2, n-1}$ the Plücker coordinates in $A_{2, n-1}$. As $\mathfrak{v}_{S}\left(\bar{p}_{i j}\right)=\left(0,0, \mathfrak{v}_{S^{\prime}}\left(\hat{p}_{i j}\right)\right)$ for $i, j<n$ we deduce $\operatorname{in}_{C_{S}}\left(R_{i, j, k, l}\right)=\operatorname{in}_{M_{S}}\left(R_{i, j, k, l}\right)$ with $i, j, k, l<n$ by induction. Consider a Plücker relation of form $R_{i, j, k, n}$ and compute

$$
\mathfrak{v}_{S}\left(\bar{p}_{l n}\right)= \begin{cases}\left(1,0, \mathfrak{v}_{S^{\prime}}\left(\hat{p}_{r i_{n}}\right)\right), & \text { if } l \neq i_{n} \\ \left(0,1, \mathfrak{v}_{S^{\prime}}\left(\hat{p}_{i_{n} j_{n}}\right)\right), & \text { if } l=i_{n}\end{cases}
$$

As $\left(i_{n}, n\right)$ is a cherry in $T_{S}$ we observe that the associated weight vector $w_{T_{S}} \in C_{S}^{\circ} \subset \operatorname{trop}\left(\operatorname{Gr}\left(2, \mathbb{C}^{n}\right)\right)$ satisfies $\left(w_{T_{S}}\right)_{l n}=\left(w_{T_{S}}\right)_{l i_{n}}=\left(w_{T_{S^{\prime}}}\right)_{l i_{n}}-1$. In particular, for $i, j, k \neq i_{n}$ we have by induction $\operatorname{in}_{M_{S}}\left(R_{i, j, k, n}\right)=\operatorname{in}_{C_{S}}\left(R_{i, j, k, n}\right)$. The only relations left to consider are of form $R_{i_{n}, j, k, n}$ for $j, k \in$ $[n-1] \backslash\left\{i_{n}\right\}$. For $M_{S}$ we compute by the above

$$
\mathfrak{v}_{S}\left(\bar{p}_{i_{n} j} \bar{p}_{k n}\right)=\mathfrak{v}_{S}\left(\bar{p}_{i_{n} k} \bar{p}_{j n}\right) \succ_{\Psi} \mathfrak{v}_{S}\left(\bar{p}_{i_{n} n} \bar{p}_{j k}\right) .
$$

Hence, $\operatorname{in}_{M_{S}}\left(R_{i_{n}, j, k, n}\right)=p_{i_{n} j} p_{k n}-p_{i_{n} k} p_{j n}$. As $\left(i_{n}, n\right)$ labels a cherry in $T_{S}$ we also have $\operatorname{in}_{C_{S}}\left(R_{i_{n}, j, k, n}\right)=$ $\operatorname{in}_{M_{S}}\left(R_{i_{n}, j, k, n}\right)$.

Theorem 2. For every iterated sequence $S$ we have $\operatorname{gr}_{S}\left(A_{2, n}\right) \cong \mathbb{C}\left[p_{i j}\right]_{i<j} / \operatorname{in}_{C_{S}}\left(\mathcal{I}_{2, n}\right)$. Conversely, for every maximal prime cone $C \subset \operatorname{trop}\left(\operatorname{Gr}\left(2, \mathbb{C}^{n}\right)\right)$ there exists a birational sequence $S$, such that $\mathbb{C}\left[p_{i j}\right]_{i<j} / \operatorname{in}_{C}\left(\mathcal{I}_{2, n}\right) \cong \operatorname{gr}_{S}\left(A_{2, n}\right)$.

Proof. Let $S$ be an iterated sequence. By Corollary 1 we can apply [Bos20, Theorem 1] and get

$$
\operatorname{gr}_{S}\left(A_{2, n}\right) \cong \mathbb{C}\left[p_{i j}\right]_{i<j} / \operatorname{in}_{M_{S}}\left(\mathcal{I}_{2, n}\right) \stackrel{\text { Proposition } 1}{=} \mathbb{C}\left[p_{i j}\right]_{i<j} / \operatorname{in}_{C_{S}}\left(\mathcal{I}_{2, n}\right) \text {. }
$$

For the second we pursue by induction: for $n=3$ there is only one maximal cone $C \subset \operatorname{trop}\left(\operatorname{Gr}\left(2, \mathbb{C}^{3}\right)\right)$. The sequence $S=\left(\varepsilon_{1}-\varepsilon_{3}, \varepsilon_{2}-\varepsilon_{3}\right)$ satisfies $C_{S}=C$. Assume the claim is true for $n-1$ and consider a maximal cone $C \subset \operatorname{trop}\left(\operatorname{Gr}\left(2, \mathbb{C}^{n}\right)\right)$ with associated tree $T_{C}$. Let $\mathrm{T}_{C}$ denote the underlying unlabeled tree. Find a cherry in $\mathrm{T}_{C}$ and remove it. Denote by $\mathrm{T}_{C}^{\prime}$ the obtained tree with $n-1$ leaves. By induction there exist an iterated sequence $S^{\prime}$ for $\operatorname{Gr}\left(2, \mathbb{C}^{n-1}\right)$ with associated tree $T_{S^{\prime}}$ of shape $\mathrm{T}_{C}^{\prime}$. Now add back the cherry and label the additional leaf by $n$. The cherry is labeled by some $i<n$ and $n$. Hence, $S:=\left(\varepsilon_{i}-\varepsilon_{n}, \varepsilon_{j}-\varepsilon_{n}, S^{\prime}\right)$ for arbitrary $j<n$ with $i \neq j$ is an iterated sequence with $T_{S}$ of shape $\mathrm{T}_{C}$ and therefore $\operatorname{gr}_{S}\left(A_{2, n}\right) \cong \mathbb{C}\left[p_{i j}\right]_{i<j} / \operatorname{in}_{C}\left(\mathcal{I}_{2, n}\right)$.

For an iterated sequence $S$ for $\operatorname{Gr}\left(2, \mathbb{C}^{n}\right)$ denote by $\mathrm{T}_{i}^{S}$ the (unlabeled) trivalent tree underlying the labeled trivalent tree $T_{i}^{S}$ with $i$ leaves in the tree sequence $\mathbb{T}_{S}$. Algorithm 1 provides a tool for comparing whether two iterated sequences induce isomorphic flat toric degenerations. Construct $\mathbb{T}_{S_{1}}, \mathbb{T}_{S_{2}}$ for two such sequences $S_{1}, S_{2}$ and consider $\mathrm{T}_{n}^{S_{1}}$ and $\mathrm{T}_{n}^{S_{2}}$. If $\mathrm{T}_{n}^{S_{1}}$ and $\mathrm{T}_{n}^{S_{2}}$ coincide then

$$
\operatorname{in}_{T_{n}^{S_{1}}}\left(\mathcal{I}_{2, n}\right) \cong \operatorname{in}_{T_{n}^{S_{2}}}\left(\mathcal{I}_{2, n}\right) .
$$

Let $\mathcal{H}_{d}:=\operatorname{conv}\left(e \in\{0,1\}^{d}\right)$ be the hypercube in $\mathbb{R}_{\geq 0}^{d}$.

Corollary 2. For every iterated sequence $S$ for $\operatorname{Gr}\left(2, \mathbb{C}^{n}\right)$ the Newton-Okounkov polytope has $\left(\begin{array}{l}n \\ 2\end{array}\right)$ vertices of form $\mathfrak{v}_{S}\left(\bar{p}_{i j}\right)$ for $1 \leq i<j \leq n$. Further, it has no interior lattice points and satisfies

$$
\Delta\left(A_{2, n}, \mathfrak{v}_{S}\right) \subset \mathcal{H}_{d}
$$


Moreover, if $S_{1}, S_{2}$ are two different iterated sequences for $\operatorname{Gr}\left(2, \mathbb{C}^{n}\right)$ with $\mathrm{T}_{n}^{S_{1}}=\mathrm{T}_{n}^{S_{2}}$, then the NewtonOkounkov polytopes are unimodularly equivalent ${ }^{4}$.

Proof. By Theorem 2 the value semi-group $S\left(A_{2, n}, \mathfrak{v}_{S}\right)$ is generated by $\left\{\mathfrak{v}_{S}\left(\bar{p}_{i j}\right)\right\}_{i<j}$. This implies $\Delta\left(A_{2, n}, \mathfrak{v}_{S}\right)=\operatorname{conv}\left(\mathfrak{v}\left(\bar{p}_{i j}\right)\right)_{i<j}$. Observe from the proof of Proposition 1 that $\mathfrak{v}_{S}\left(\bar{p}_{i j}\right) \in\{0,1\}^{d}$. Therefore, $\mathfrak{v}_{S}\left(\bar{p}_{i j}\right) \notin \operatorname{conv}\left(\mathfrak{v}_{S}\left(\bar{p}_{k l}\right) \mid(k<l) \neq(i<j)\right)$. Hence, for all $1 \leq i<j \leq n$ every $\mathfrak{v}_{S}\left(\bar{p}_{i j}\right)$ is indeed a vertex and $\Delta\left(A_{2, n}, \mathfrak{v}_{S}\right) \subset \mathcal{H}_{d}$.

Regarding the second statement, let $S_{1}, S_{2}$ be two iterated sequences. Assume we have $\mathrm{T}_{n}^{S_{1}}=\mathrm{T}_{n}^{S_{2}}$ and consider the lattice ideals associated to each $\operatorname{in}_{C_{S_{i}}}\left(\mathcal{I}_{2, n}\right)$ (see e.g. [BLMM17, Lemma 2]). The corresponding lattice points $\left\{l_{j}^{i}\right\}_{j=1, \ldots,\left(\begin{array}{c}n \\ 2\end{array}\right)}$ define polytopes in their ambient lattice which are unimodularly equivalent to $\Delta\left(A_{2, n}, \mathfrak{v}_{S_{i}}\right)$ (see [BLMM17, proof of Theorem 4] for the precise construction). Therefore, (4.2) implies $\Delta\left(A_{2, n}, \mathfrak{v}_{S_{1}}\right) \cong \Delta\left(A_{2, n}, \mathfrak{v}_{S_{2}}\right)$.

Given a maximal cone $C_{T} \in \operatorname{trop}\left(\operatorname{Gr}\left(2, \mathbb{C}^{n}\right)\right)$ with associated labeled trivalent tree $T$ the rays of $C$ are in bijection with internal edges of $T$, i.e. those not adjacent to any leaf. Internal edges give rise to partitions of $[n]$ into two sets (each of cardinality $\geq 2$ ) corresponding to the labelings of the leaves on either side of the edge. Given a partition $(A, B)$ of $[n]$ the ray is $E_{A, B}=-\sum_{i \in A} \sum_{j \in B} e_{i j} \in \mathbb{R}\left(\begin{array}{l}n \\ 2\end{array}\right)$, see [SS04, page 396].

Corollary 3. Let $S$ be an iterated sequence $S$ for $\operatorname{Gr}\left(2, \mathbb{C}^{n}\right)$ and for $n \geq 4$ we consider the projection $\pi_{n}: \mathbb{R}^{\left(\begin{array}{c}n \\ 2\end{array}\right)} \rightarrow \mathbb{R}^{\left(\begin{array}{c}n-1 \\ 2\end{array}\right)}$ onto all coordinates that do not involve $n$. Then $\mathbb{T}_{S}=\left(T_{3}, T_{4}^{S}, \ldots, T_{n}^{S}\right)$ corresponds to a sequence of cones $\left(C_{T_{3}}, C_{T_{4}^{S}}, \ldots, C_{T_{n}^{S}}\right)$ with $C_{T_{i}^{S}} \in \operatorname{trop}\left(\operatorname{Gr}\left(2, \mathbb{C}^{i}\right)\right)$ and $\pi_{i}\left(C_{T_{i}^{S}}\right)=C_{T_{i-1}^{S}}$.

Proof. The first property follows from Theorem 2. For the second notice that if $(A, B)$ is a partition of

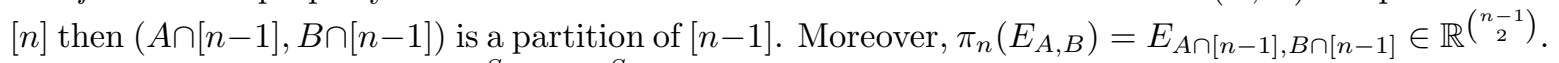
For every $4 \leq i \leq n$ the trees $T_{i}^{S}$ and $T_{i-1}^{S}$ have all interior edges in common, except the one adjacent to the cherry added when constructing $T_{i}^{S}$ from $T_{i-1}^{S}$. Hence, the rays of $C_{T_{i}^{S}}$ are mapped to the rays of $C_{T_{i-1}^{S}}$ by $\pi_{n}$.

Remark 3. In [KM19, Proposition 8.16] the authors describe an initial ideal $\operatorname{in}_{M}(I)$ for an ideal $I \subset \mathbb{C}\left[x_{1} \ldots, x_{n}\right]$ with respect to a matrix $M \in \mathbb{R}^{n \times d}$ as $\operatorname{in}_{u_{1}}\left(\ldots \operatorname{in}_{u_{d}}(I) \ldots\right)$, where $u_{1}, \ldots, u_{d}$ are the columns of $M$. This leads to a sequence of initial ideals of $I$, namely $I, \operatorname{in}_{u_{d}}(I), \operatorname{in}_{u_{d-1}}\left(\operatorname{in}_{u_{d}}(I)\right)$ and so on. The sequence of cones $\left(C_{T_{3}}, C_{T_{4}^{S}}, \ldots, C_{T_{n}^{S}}\right)$ from Corollary 3 also yields a sequence of initial ideals $\operatorname{in}_{T_{3}}\left(\mathcal{I}_{2,3}\right), \operatorname{in}_{T_{4}^{S}}\left(\mathcal{I}_{2,4}\right), \ldots, \operatorname{in}_{T_{n}^{S}}\left(\mathcal{I}_{2, n}\right)$. This sequence is different in nature from the first as we are taking initial ideals of different ideals.

The following definition allows us to interpret iterated sequences for $\operatorname{Gr}\left(2, \mathbb{C}^{n}\right)$ in a combinatorial way in Corollary 4 below.

Definition 14. The tree graph $\mathcal{T}$ is an infinite graph whose vertices at level $i \geq 3$ correspond to trivalent trees with $i$ leaves. There is an arrow $\mathrm{T} \rightarrow \mathrm{T}^{\prime}$, if $\mathrm{T}$ has $i$ leaves, $\mathrm{T}^{\prime}$ has $i+1$ leaves and $\mathrm{T}^{\prime}$ can be obtained from $\mathrm{T}$ by attaching a new boundary edge to the middle of some edge of $\mathrm{T}$. There is a unique source $\mathrm{T}_{3}$ at level 3 (see Figure 1).

Corollary 4. Every iterated sequence $S$ for $\operatorname{Gr}\left(2, \mathbb{C}^{n}\right)$ corresponds to a path from $\mathrm{T}_{3}$ to $\mathrm{T}_{n}^{S}$ in the tree graph $\mathcal{T}$.

Proof. The underlying unlabeled trees in the sequence $\mathbb{T}_{S}=\left(T_{3}, T_{4}^{S}, \ldots, T_{n}^{S}\right)$ associated to $S$ define the path $\mathrm{T}_{3} \rightarrow \mathrm{T}_{4}^{S} \rightarrow \cdots \rightarrow \mathrm{T}_{n}^{S}$ in $\mathcal{T}$.

\footnotetext{
${ }^{4}$ Two polytopes $P, Q \subset \mathbb{R}^{d}$ are called unimodularly equivalent if there exists matrix $M \in G L_{d}(\mathbb{Z})$ and $w \in \mathbb{Z}^{d}$ $Q=f_{M}(P)+w$, where $f_{M}(x)=x M$ for $x \in \mathbb{R}^{d}$. It is denoted $Q \cong P$ and by [CLS11, $\$ 2.1$ and $\S 2.3$ ] implies that the associated projective toric varieties are isomorphic.
} 


\section{$5 \quad$ References}

[AB04] Valery Alexeev and Michel Brion. Toric degenerations of spherical varieties. Selecta Math. (N.S.), 10(4):453478, 2004.

[And13] Dave Anderson. Okounkov bodies and toric degenerations. Math. Ann., 356(3):1183-1202, 2013.

$\left[\mathrm{BFF}^{+} 18\right]$ Lara Bossinger, Xin Fang, Ghislain Fourier, Milena Hering, and Martina Lanini. Toric degenerations of $\operatorname{Gr}(2, n)$ and $\operatorname{Gr}(3,6)$ via plabic graphs. Ann. Comb., 22(3):491-512, 2018.

[BG09] Winfried Bruns and Joseph Gubeladze. Polytopes, rings, and K-theory. Springer Monographs in Mathematics. Springer, Dordrecht, 2009.

[BHV01] Louis J. Billera, Susan P. Holmes, and Karen Vogtmann. Geometry of the space of phylogenetic trees. Adv. in Appl. Math., 27(4):733-767, 2001.

[BLMM17] Lara Bossinger, Sara Lamboglia, Kalina Mincheva, and Fatemeh Mohammadi. Computing toric degenerations of flag varieties. In Combinatorial algebraic geometry, volume 80 of Fields Inst. Commun., pages 247-281. Fields Inst. Res. Math. Sci., Toronto, ON, 2017.

[Bos18] Lara Bossinger. Toric degenerations: a bridge between representation theory, tropical geometry and cluster algebras. PhD thesis, Universität zu Köln, 2018.

[Bos20] Lara Bossinger. Full-rank valuations and toric initial ideals. Int. Math. Res. Not., (rnaa071), 2020.

[Cal02] Philippe Caldero. Toric degenerations of Schubert varieties. Transform. Groups, 7(1):51-60, 2002.

[CLS11] David A. Cox, John B. Little, and Henry K. Schenck. Toric varieties. American Mathematical Soc., 2011.

[Eis95] David Eisenbud. Commutative algebra, volume 150 of Graduate Texts in Mathematics. Springer-Verlag, New York, 1995. With a view toward algebraic geometry.

[FFL11] Evgeny Feigin, Ghislain Fourier, and Peter Littelmann. PBW filtration and bases for irreducible modules in type $A_{n}$. Transform. Groups, 16(1):71-89, 2011.

[FFL17] Xin Fang, Ghislain Fourier, and Peter Littelmann. Essential bases and toric degenerations arising from birational sequences. Adv. Math., 312:107-149, 2017.

[GL96] Nicolae Gonciulea and Venkatramani Lakshmibai. Degenerations of flag and Schubert varieties to toric varieties. Transform. Groups, 1(3):215-248, 1996.

[KK12] Kiumars Kaveh and Askold G. Khovanskii. Newton-Okounkov bodies, semigroups of integral points, graded algebras and intersection theory. Ann. of Math. (2), 176(2):925-978, 2012.

[KM19] Kiumars Kaveh and Christopher Manon. Khovanskii bases, higher rank valuations, and tropical geometry. SIAM J. Appl. Algebra Geom., 3(2):292-336, 2019.

[LB15] Venkatramani Lakshmibai and Justin Brown. The Grassmannian variety, volume 42 of Developments in Mathematics. Springer, New York, 2015. Geometric and representation-theoretic aspects.

[LM09] Robert Lazarsfeld and Mircea Mustaţă. Convex bodies associated to linear series. Ann. Sci. Éc. Norm. Supér. (4), 42(5):783-835, 2009.

[MS15] Diane Maclagan and Bernd Sturmfels. Introduction to tropical geometry, volume 161 of Graduate Studies in Mathematics. American Mathematical Society, Providence, RI, 2015.

[RW19] K. Rietsch and L. Williams. Newton-Okounkov bodies, cluster duality, and mirror symmetry for Grassmannians. Duke Math. J., 168(18):3437-3527, 2019.

[SS04] David Speyer and Bernd Sturmfels. The tropical Grassmannian. Adv. Geom., 4(3):389-411, 2004.

Lara Bossinger

Universidad Nacional Autónoma de México

Instituto de Matemáticas Unidad Oaxaca

León 2, altos, Oaxaca de Juárez, Centro Histórico

68000 Oaxaca, México

Email: lara@im.unam.mx 